\title{
Infections and foreign bodies in ENT
}

\author{
Peter Kullar, MA MRCS (ENT) [Specialty Trainee in Otolaryngology] and Philip D Yates, \\ FRCS (ORL-HNS) [Consultant Otolaryngologist] \\ Freeman Hospital, Newcastle upon Tyne, UK
}

\begin{abstract}
Infections play a major role in the practice of ENT. Microbial penetration into tissues of the head and neck can initiate a focal reaction causing superficial self-resolving infections. However, some of these have the potential to develop into life-threatening disease. We provide an overview of the most common ENT infections with focus on the presentation, diagnosis and management. Foreign bodies of the ear, nose and throat are a common presentation to primary and emergency care. Most commonly these are seen in children and include plastic toys, beads and foodstuffs inserted into the ears and nose. Diagnosis is often delayed as insertion is usually not witnessed. In exceptional cases airway foreign bodies can present as a life-threatening emergency. Removal of foreign bodies can usually be achieved by a skilled practitioner with minimal complications. Methods of removal include suction catheters, syringing, and use of instrumentation. In adults, the treatment of oesophageal food bolus obstruction may require a combination of medical and surgical intervention.
\end{abstract}

\section{Keywords}

Acute otitis externa; acute otitis media; foreign bodies; mastoiditis; periorbital cellulitis; rhinosinusitis; supraglottitis; tonsillitis

\section{Infections of the external ear}

\section{Otitis externa}

Acute otitis externa (AOE) is a common infection which presents with pain, itch and discharge. Classically, the pain is exacerbated by traction on the pinna, distinguishing it from the pain associated with acute otitis media. Initially there will be inflammation of the skin of the external auditory canal (EAC), but if the infection progresses then the canal skin becomes oedematous leading to narrowing of the canal lumen. This can lead to a buildup of debris in the canal (Figure 1a). AOE may be idiopathic, secondary to ear canal trauma or related to exposure of the ear canal to water and can therefore be related to swimming. Common causative organisms are Pseudomonas aeruginosa, Proteus, coliforms and staphylococci. AOE usually resolves with treatment but may progress to become a chronic infection. Patients who go on to develop chronic otitis externa sometimes have an underlying dermatological condition such as eczema or psoriasis. Chemical irritants such as hair dyes,

Conflicts of interest: none declared. 
or even topical preparations used to treat the condition, can be a factor. The mainstay of treatment of otitis externa is by clearing the ear canal of debris, by microsuction or gentle syringing, and the application of topical antibiotic \pm steroid preparations. However, if antibiotic drops are prescribed for a prolonged period this may predispose to fungal infection which may need treatment with an anti-fungal such as topical nystatin (Figure 1b).

Sometimes it is not possible to instil ear drops into the canal due to canal wall oedema. If this is the case then the ear canal should be cleared with gentle microsuction and an aural wick inserted to allow application of topical treatment to the canal wall skin. ${ }^{1}$ AOE can result in spreading cellulitis of the pinna (Figure 1c), AOE can be differentiated from auricular perichondritis, a diffuse inflammatory although not necessarily infectious process, by its involvement of the ear lobe. The most significant, although uncommon, complication of ear canal infection is malignant otitis externa. This is a misnomer as the condition is not neoplastic in nature but is a spreading osteomyelitis of the skull base usually caused by $P$. aeruginosa infection. It is most commonly seen in elderly diabetics and the immunocompromised. It presents with severe pain, discharge and granulation tissue in the floor of the ear canal. If left untreated it leads to multiple lower cranial nerve palsies and is potentially fatal. Treatment consists of repeated clearance of the EAC and at least six weeks of intravenous anti-pseudomonal antibiotics.

Furuncles are pustular lesions surrounding a hair follicle most often located at the inferior aspect of the entrance to the EAM (Figure 1d). Furunculosis is exquisitely painful and treatment is by incision and drainage under local anaesthesia.

\section{Herpes zoster oticus}

Herpes zoster oticus is caused by varicella zoster virus which infects and then remains latent in dorsal root ganglia. Reactivation causes a vesicular eruption along the dermatome of affected nerves. The first symptom is usually otalgia followed by blistering of the concha. Ramsay Hunt syndrome results when there is an associated lower motor neurone facial palsy due to involvement of the facial nerve. Management consists of analgesia, aural toilet, oral acyclovir and prednisolone.

\section{Infections of the middle ear}

\section{Acute otitis media (AOM)}

$\mathrm{AOM}$ is one of the most common infectious diseases seen in children, with a peak incidence in the first two years of life. Although viral infection is important in the pathogenesis of AOM, the majority of cases will develop subsequent bacterial colonization, and hence AOM should be considered a predominantly bacterial infection. The organisms most commonly responsible for AOM are Streptococcus pneumoniae, Haemophilus influenzae and Moraxella catarrhalis.

Older children will usually complain of earache, whereas infants become irritable and pull at the affected ear. A high fever is often present and may be associated with systemic symptoms of infection. Otoscopy classically shows a thickened hyperaemic tympanic membrane (Figure 2a). Further progression may lead to spontaneous rupture of the tympanic 
membrane resulting in otorrhoea (Figure $2 \mathrm{~b}$ ). If recurrent $\mathrm{AOM}$ occurs along with recurrent infections in other body systems an underlying immune deficiency should be considered.

The need for antibiotic treatment is a subject of controversy. There are several large studies that suggest the outcome of AOM is not affected by prescribing oral antibiotic and the majority of cases settle without treatment. In the UK there is a consensus view that there is no need for antibiotics in the first 48 hours, unless the child is systemically unwell or very young (when the diagnosis may be less certain). If antibiotic treatment is considered necessary then amoxicillin remains the first-line treatment. In resistant cases amoxicillin should be given in combination with clavulanate. ${ }^{2}$

Although the vast majority of cases of AOM resolve spontaneously, it can result in more serious complications due to spread of infection into adjacent structures. These can either be intra-temporal (mastoiditis, petrositis, labyrinthitis and facial paralysis) or intracranial (meningitis, lateral sinus thrombosis and intracranial abscesses).

\section{Mastoiditis}

If pus collects in the mastoid air cells under pressure, necrosis of the bony trabeculae occurs resulting in the formation of an abscess cavity. Infection may then progress to periostitis and subperiosteal abscess, or to more serious intracranial infection. The most common indication that acute mastoiditis is developing is pain and tenderness over the mastoid process. As the infection progresses oedema and erythema of the postauricular soft tissues with loss of the postauricular crease develops. This results in anteroinferior displacement of the pinna (Figure $2 \mathrm{c}$ and $\mathrm{d}$ ). If a subperiosteal abscess has developed fluctuance may be elicited in the postauricular area. Rarely, pus extends into the neck from the medial aspect of the mastoid tip (Bezold's abscess). Once the diagnosis of acute mastoiditis is suspected the radiological investigation of choice is a CT scan. This helps determine whether a subperiosteal abscess has developed and identifies the presence of intracranial complications.

Uncomplicated acute mastoiditis can be successfully managed by antibiotic therapy alone, but a certain number of patients will require surgical intervention. When there is no clinical or radiological indication of subperiosteal abscess or intracranial extension of disease then high-dose intravenous antibiotics should be commenced. If after 24 hours of treatment there has been improvement in the patient's condition then it is likely that surgery will not be required. However, if there is no evidence of resolution, or if symptoms progress, a cortical mastoidectomy should be performed. In those cases in which subperiosteal abscess or intracranial extension of disease are suspected, surgery in combination with high-dose intravenous antibiotics should be the first-line therapy. ${ }^{3}$

\section{Chronic suppurative otitis media (CSOM)}

CSOM is persistent discharge through an infected perforated tympanic membrane or from an infected cholesteatoma.

A perforated tympanic membrane (Figure 2e) is prone to persistent or recurrent infection. Otorrhoea and hearing loss are usually the only symptoms. The discharge is typically mucopurulent as it is arising from the middle ear mucosa. Pain is not usually a feature. 
Treatment with topical antibiotics will usually resolve the discharge but recurrently infected perforations are best treated by surgical repair.

A cholesteatoma is caused by accumulation of desquamated epithelium in a retraction of the tympanic membrane (Figure 2f). Squamous epithelium that would normally migrate out of the ear canal gets trapped in the pocket and acts as a focus for infection, usually with $P$. aeruginosa. This causes the classic symptom of smelly otorrhoea. As the pocket expands it erodes the ossicles leading to a conductive hearing loss. If left untreated a cholesteatoma can lead to more serious complications such as facial nerve palsy and life-threatening intracranial infection. For this reason a cholesteatoma should always be removed surgically via a mastoidectomy, unless the patient is a significant anaesthetic risk due to other morbidity.

\section{Infections of the nose and paranasal sinuses}

\section{Rhinosinusitis}

The term rhinitis is used to describe inflammation of the nasal mucosa and sinusitis to describe inflammation of the sinus mucosa. In reality most infections affect the nose and paranasal sinuses and are referred to as rhinosinusitis. Rhinosinusitis is divided into acute (symptoms for less than 12 weeks) or chronic (symptoms for more than 12 weeks). ${ }^{4}$

Viral rhinosinusitis (common cold) is extremely common. Acute rhinosinusitis complicates $0.5-5 \%$ of viral upper respiratory tract infections. It develops due to blockage of the natural sinus ostium preventing efficient drainage. This can be due to the inflammatory response, underlying anatomical abnormality or a combination of both. The most commonly isolated bacteria are $S$. pneumoniae, $H$. influenzae. The patient will initially have symptoms of nasal obstruction and discharge, but as infection in the sinus develops facial pain, which is often worsen on bending forward, will become a prominent symptom. Endoscopic examination of the nasal cavity will reveal pus arising from the sinuses, usually in the middle meatus (Figure 3a).

The mainstay of treatment for acute sinusitis is to establish drainage of the sinuses by decongestants, saline nasal irrigation and humidification. Antibiotics are only recommended for sinusitis that fails to improve within 48 hours. Co-amoxiclav or quinolones are most often prescribed. Further investigation by CT scanning is only indicated in cases that develop complications or patients who go on to have surgical treatment for chronic rhinosinusitis.

Chronic rhinosinusitis is typified by ongoing symptoms and should be treated with antimicrobials covering both aerobic and anaerobic bacteria, for example, co-amoxiclav or a macrolide. A prolonged course of antibiotics for at least 2 weeks is often required. Nasal and systemic steroids are also used to decrease the inflammatory response. Surgical management is warranted after failure of maximal medical treatment with the aim of enlarging the natural sinus ostia to enhance drainage and restore natural mucociliary clearance. 


\section{Periorbital cellulitis}

The commonest complication of acute rhinosinusitis is periorbital cellulitis (Figure 3b) which is a result of infection spreading from the ethmoid sinuses into the periorbital tissue. Initially only the lid may be affected (preseptal cellulitis) but if the infection progresses oedema and abscess formation in the orbit leads to chemosis and proptosis. Once an abscess has formed and the eye becomes proptosed there is a significant risk to vision and the abscess should be surgically drained on an urgent basis. ${ }^{5}$

\section{Infections of the oropharynx}

The majority of oropharyngeal infections are caused by viruses (80-90\%). Bacterial infections are likely to present with more severe local and systemic symptoms. Most cases of pharyngitis require symptomatic treatment only.

Acute tonsillitis can be caused by viruses or bacteria (typically Group A Streptococcus). Clinical differentiation between viral and bacterial infection is difficult. Patients present similarly with odynophagia, fever, malaise and cervical lymphadenopathy. The palatine tonsils are often enlarged and may be covered with exudates, either in follicles or covering the whole of the tonsils (Figure 4). An important differential diagnosis is infectious mononucleosis which is caused by the Epstein-Barr virus. The severity of this condition is variable from mild, lasting just a few days with minor symptoms, to severe with systemic complications such as hepatosplenomegaly.

Analgesia and fluids is the mainstay of treatment of pharyngeal infections. If bacterial tonsillitis is suspected, antibiotics are useful and penicillin is considered the antibiotic of choice. Patients can be managed on an outpatient basis but may need admission if unable to sustain nutrition or for uncontrollable pain. Recurrent tonsillitis is an indication for tonsillectomy (see SIGN guidelines). ${ }^{6}$

Peritonsillar abscess is a complication of tonsillitis caused by spread of infection into the peritonsillar space which lies between the capsule of the tonsil and the pharyngeal muscles. The affected tonsil is pushed medially by the abscess and the patient often has trismus. Treatment is by transoral aspiration or incision and drainage of the abscess along with antibiotic therapy.

\section{Supraglottitis (epiglottitis)}

Although the term epiglottis is frequently used for this condition, it is more correctly called supraglottitis, as cellulitis involves multiple areas of the supraglottis. Typically, acute supraglottitis presents in children between the ages of 2 and 6 years, although any age group, including adults, can be affected. $H$. influenzae type B (HiB) is the responsible pathogen in the majority of cases and, as a result of the introduction of the HiB vaccine, the incidence of supraglottitis has been reduced by more than $90 \%$. Although supraglottitis is a rare infection awareness of the disease is important due the risk of airway obstruction and death (particularly in children) if not promptly diagnosed and treated. 
Acute supraglottitis in children is a rapidly progressive disease and symptoms will usually only have been present for a matter of hours at presentation. The typical features are pyrexia, difficulty in breathing and severe odynophagia, resulting in drooling. The child is usually irritable, sitting or leaning forward and the voice is typically muffled. Inspiratory stridor is a late feature occurring when the airway is almost completely obstructed.

Once the diagnosis of supraglottitis is suspected further investigations should not be undertaken in children as any procedures that induce anxiety in the patient, including intraoral examination and Venipuncture, may precipitate complete airway obstruction.

The management of a child with suspected supraglottitis requires close cooperation between the otolaryngologist, anaesthetist and paediatrician. The child should be directly transferred to theatre where equipment for emergency tracheostomy must be available. After inhalational anaesthesia the supraglottis can be inspected and the presence of erythema and oedema confirms the diagnosis. The airway is then secured by endotracheal intubation. Once the airway is safe blood cultures and swabs of the supraglottis can be obtained and an intravenous cannula inserted. Parenteral antibiotic therapy should then be started. Supraglottitis usually responds rapidly and extubation is often possible after 48-72 hours.

In adults airway compromise is rare due to the larger size of the larynx. The diagnosis can be made by flexible nasendoscopy and appropriate treatment commenced. Only a small proportion will require endotracheal intubation. ${ }^{7}$

\section{Foreign bodies}

\section{Foreign bodies in the airway}

Airway foreign bodies are most common in patients under the age of 15 , with the highest incidence between 1 and 3 years. Foreign body aspiration results in approximately 3000 deaths in US every year. Younger children are more prone to airway foreign bodies due to immature glottic closure and laryngeal elevation during swallowing. Vegetative matter is the most common airway foreign body, particularly peanuts and seeds but inorganic items such as coins and plastic toys are also found.

Airway foreign bodies most commonly become lodged in bronchi having passed through the larynx and trachea. Larger objects impacting at the larynx can cause complete airway obstruction. Acute airway obstruction by a foreign body is an absolute emergency which will result in death if not treated immediately. For children younger than one year sharp back blows are recommended. For older children and adults the Heimlich manoeuvre can be used in an attempt to expel inhaled foreign bodies. This increases intrathoracic and upper airway pressure by the application of external abdominal compression.

Bronchial foreign bodies are most often found in the right main bronchus as it is wider and less angulated from the trachea. Aspirated foreign bodies initially cause paroxysms of coughing but reflexes then fatigue. Patients can then remain asymptomatic for weeks until complications occur, such as pneumonia and abscess formation. 
Posterior anterior and lateral soft tissue neck radiographs will identify radio-opaque foreign bodies. A foreign body causing obstruction of a bronchus may cause lung segment collapse and subsequent consolidation. In the early stages however there may be no radiological abnormality.

If an airway foreign body is suspected then bronchoscopy should be urgently performed by a practitioner skilled in using bronchoscopes and optical forceps.

\section{Foreign bodies in the pharynx}

Sharp and irregular foreign bodies, such as fish bones, can become impacted in the tongue base, vallecula, pyriform fossae and most commonly the tonsils. These can often be accurately localized by the patient.

Pharyngeal foreign bodies can be removed in the clinic. After adequate analgesia with cophenylcaine spray the tongue should be depressed with a McGill laryngoscope. Angled grasping forceps can then be used to retrieve the foreign body. If the patient cannot tolerate this then removal under general anaesthetic is required.

\section{Foreign bodies in the oesophagus}

By far the most common oesophageal foreign bodies in children are coins. Food bolus impaction is commoner in adults. Oesophageal foreign bodies often lodge in the upper oesophagus at the level of the cricopharygeus. More distal impaction may occur at the level of the aortic arch, left main bronchus or gastrooesophageal junction.

Oesophageal foreign bodies typically present with complete dysphagia.

Food bolus obstruction is often idiopathic. However, predisposing factors include mucosal strictures and importantly neoplasia. Flexible nasendoscopy is often normal although pooling in the pyriform fossae may be seen.

The initial management of food bolus obstruction is conservative. Acidic fizzy drinks and enzymatically active juices (such as pineapple) have been shown to be effective.

Antispasmodics such as buscopan and hyoscine are also routinely employed. Intravenous glucagon can be used as a lower oesophageal dilator, however, the evidence for its efficacy is questionable. ${ }^{8}$ Most oesophageal foreign bodies can be managed medically; however, disc battery ingestion requires immediate removal due to the risk of severe caustic damage to oesophageal mucosa potentially resulting in perforation and mediastinitis. If conservative management fails an oesophagoscope is passed under general anaesthesia and advanced into the oesophageal lumen until the foreign body is visualized. If a distal foreign body is suspected it can be removed or pushed through to the stomach using a flexible endoscope. In patients with an idiopathic food bolus it is prudent to perform a barium swallow at 2 weeks to exclude the possibility of an oesophageal neoplasm. ${ }^{9}$

\section{Foreign body in the ear}

The most common foreign bodies include beads, plastic toys, paper and insects (Figure 5a). In adults the most common foreign bodies are cotton wool from overzealous ear cleaning. 
Aural foreign body insertion is usually unwitnessed in children and may present as an incidental finding. Attempts by the patient or non-skilled practitioners to remove a foreign body often push it further into the canal. The main risk of attempting removal is perforation of the tympanic membrane and damage to middle ear structures. Adequate visualization, equipment and patient cooperation are the key to successful removal. In children the first attempt at removal is extremely important as removal becomes increasingly more difficult if pain is inflicted at the first attempt. Most aural foreign bodies do not require immediate removal; however batteries should be removed on an urgent basis due to a corrosive effect that can cause permanent damage to the tympanic membrane and EAC.

There are many techniques to remove foreign bodies from the ear under direct vision using an adequate head light or an operating microscope. Options include crocodile forceps, wax hooks, suction catheters and ear syringing. Irrigation should not be performed in patients with batteries in the ear. Insects can be killed by instilling mineral oil or alcohol into the ear. Acetone is useful in the removal of superglue.

\section{Foreign bodies in the nose}

Nasal foreign bodies are most commonly found in children less than 3 years of age. The most common site for nasal foreign bodies is the floor of the nose below the inferior turbinate. The initial insertion is often unwitnessed and patients may present months after the original incident with foul smelling unilateral discharge due to secondary infection. In any child presenting with a unilateral nasal discharge a foreign body should be suspected even without a corollary history. Nasal foreign bodies can be organic or inorganic and include plastic toys, beads, food, paper and foam. Nasal foreign bodies should be removed on a semi-urgent basis due to the risk of aspiration and airway compromise. Button batteries must be removed immediately as permanent damage to the nasal mucosa can occur in less than 2 hours resulting in a septal perforation and potential collapse of the nasal dorsum.

Most nasal foreign bodies can be removed in the clinic. Initially, the patient should be asked to blow their nose; this may need to be demonstrated to young children. It is also possible to generate upper airway positive pressure with the 'mother's kiss' technique. Here, a parent places their lips over that of the child forming an airtight seal and blows hard covering the unobstructed nostril with a finger.

In children it can be helpful sit them on a parent's knee swaddled in a blanket or tightly cuddled before attempting manual removal. It may be helpful initially to spray the nose with co-phenylcaine to reduce mucosal oedema. Under direct vision with good illumination most foreign bodies can be removed with a wax hook or Jobson Horne probe. The instrument should be rotated around the foreign body which can be then raked anteriorly. Forceps can be used to grasp items such as paper. In some cases a general anaesthetic will be required to remove the foreign body.

Occasionally, if foreign bodies are left in situ for prolonged periods, concretions can form around them forming a 'rhinolith' (Figure 5b). 


\section{REFERENCES}

1. Kaushik V, Malik T, Saeed SR. Interventions for acute otitis externa. Cochrane Database Syst Rev. 2010(1) Art. No.:CD004740.

2. Venekamp RP, Sanders S, Glasziou PP, Del Mar CB, Rovers MM. Antibiotics for acute otitis media in children. Cochrane Database of Syst Rev. 2013; (1) Art. No.:CD000219.

3. Psarommatis IM, Voudouris C, Douros K, Giannakopoulos P, Bairamis T, Carabinos C. Algorithmic management of pediatric acute mastoiditis. Int J Pediatr Otorhinolaryngol. 2012; 76:791-6. [PubMed: 22405736]

4. Fokkens WJ, Lund VJ, Mullol J, et al. EPOS 2012: European position paper on rhinosinusitis and nasal polyps 2012. A summary for otorhinolaryngologists. Rhinol. 2012; 50:1-12.

5. Bedwell J, Bauman NM. Management of pediatric orbital cellulitis and abscess. Curr Opin Otolaryngol Head Neck Surg. 2011; 19:467-73. [PubMed: 22001661]

6. Management of sore throat and indications for tonsillectomy. http://www.sign.ac.uk/pdf/sign117.pdf

7. Riffat F, Jefferson N, Bari N, McGuinness J. Acute supraglottitis in adults. Ann Otol Rhinol Laryngol. 2011; 120:296-9. [PubMed: 21675584]

8. Weant KA, Weant MP. Safety and efficacy of glucagon for the relief of acute esophageal food impaction. Am J Health Syst Pharm. 2012; 69:573-7. [PubMed: 22441787]

9. Rodríguez H, Passali GC, Gregori D, et al. Management of foreign bodies in the airway and oesophagus. Int J Pediatr Otorhinolaryngol. 2012; 76(suppl 1):84-91. Epub 2012 Feb 24. 

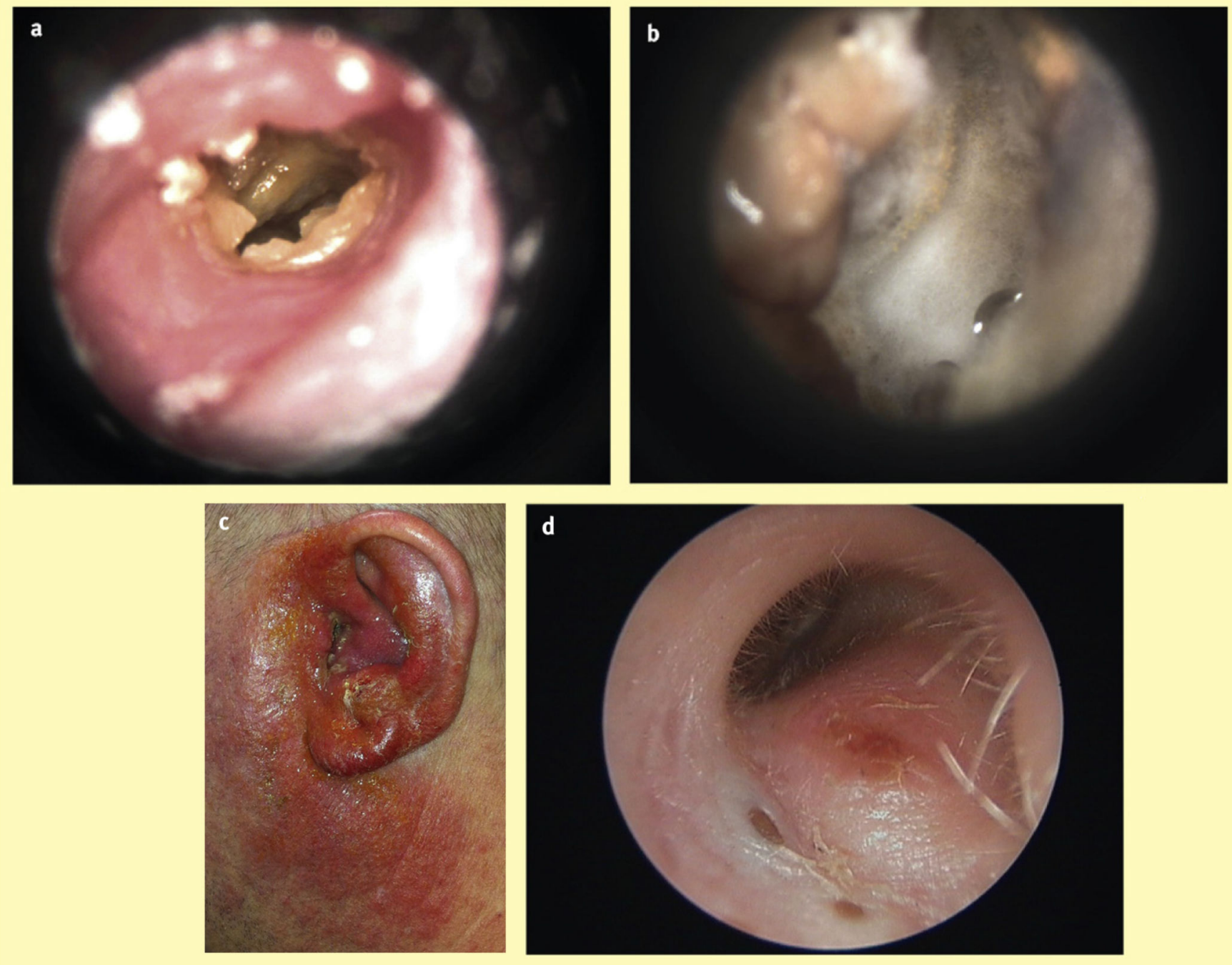

Figure 1.

(a) Acute otitis externa with erythema of the ear canal skin and debris obscuring the tympanic membrane. (b) Fungal otitis externa with fungal hyphae seen on the debris in the ear canal. (c) Spreading cellulitis of the pinna. (d) A furuncle in the floor of the ear canal. 

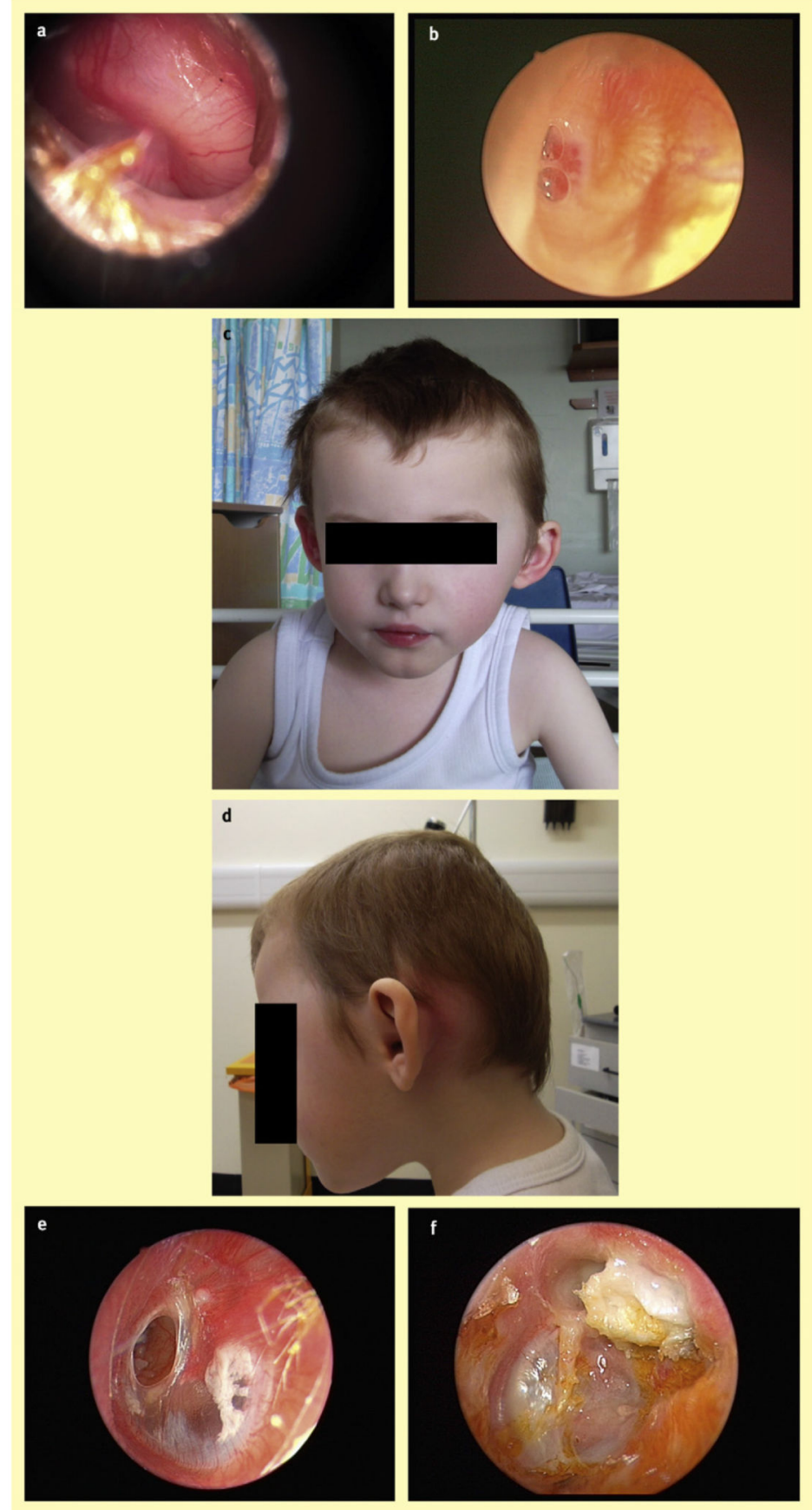

Figure 2.

(a) Acute otitis media with a bulging erythematous tympanic membrane. (b) Acute otitis media with discharge through a perforation. (c \& d) Acute left mastoiditis in a child. (e) A chronic dry perforation of a left tympanic membrane. (f) A cholesteatoma of a left ear. 

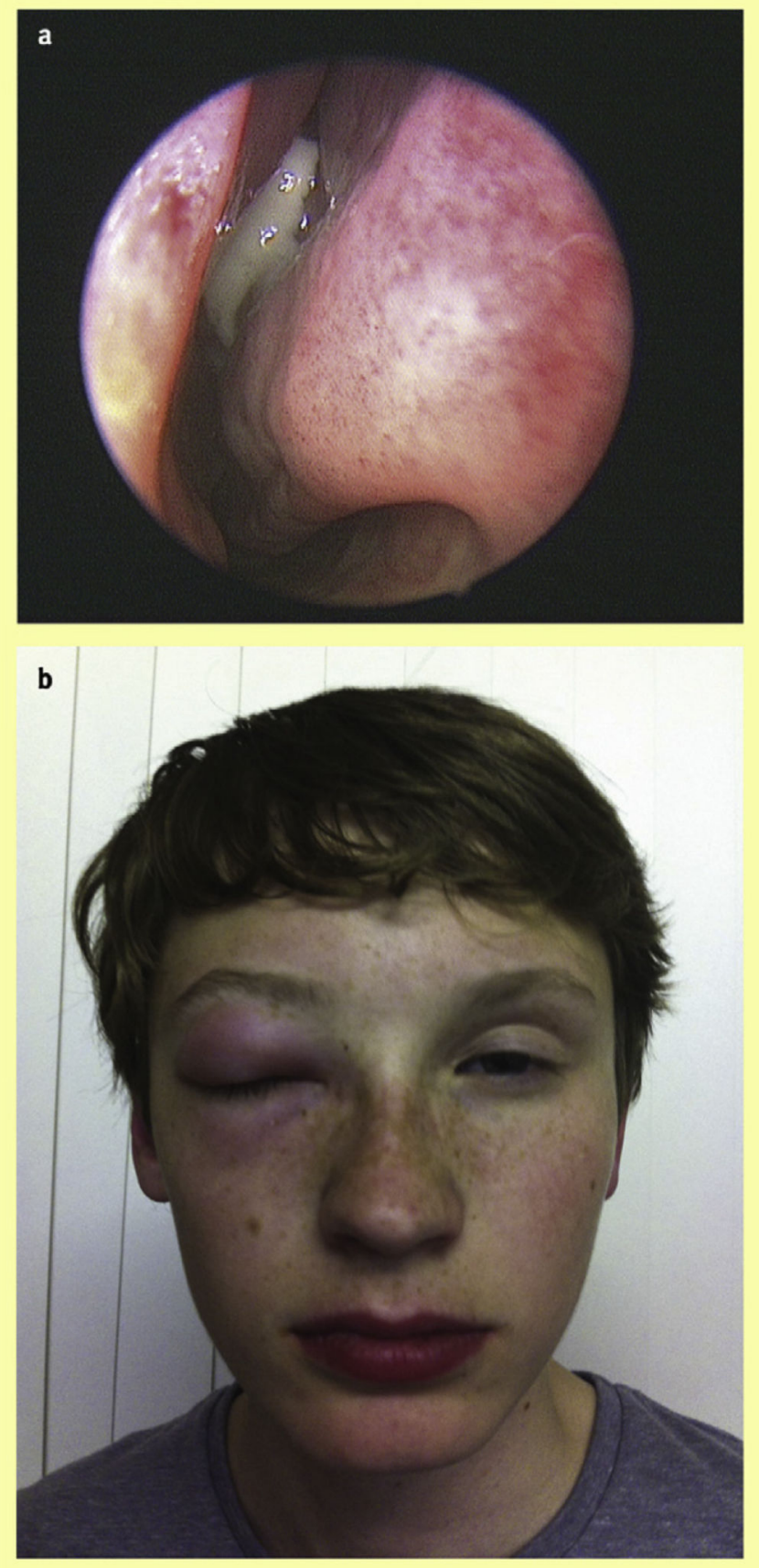

Figure 3.

(a) Endoscopic view of the left nasal cavity demonstrating pus in the middle meatus. (b) Right periorbital cellulites complicating acute rhinosinusitis. 


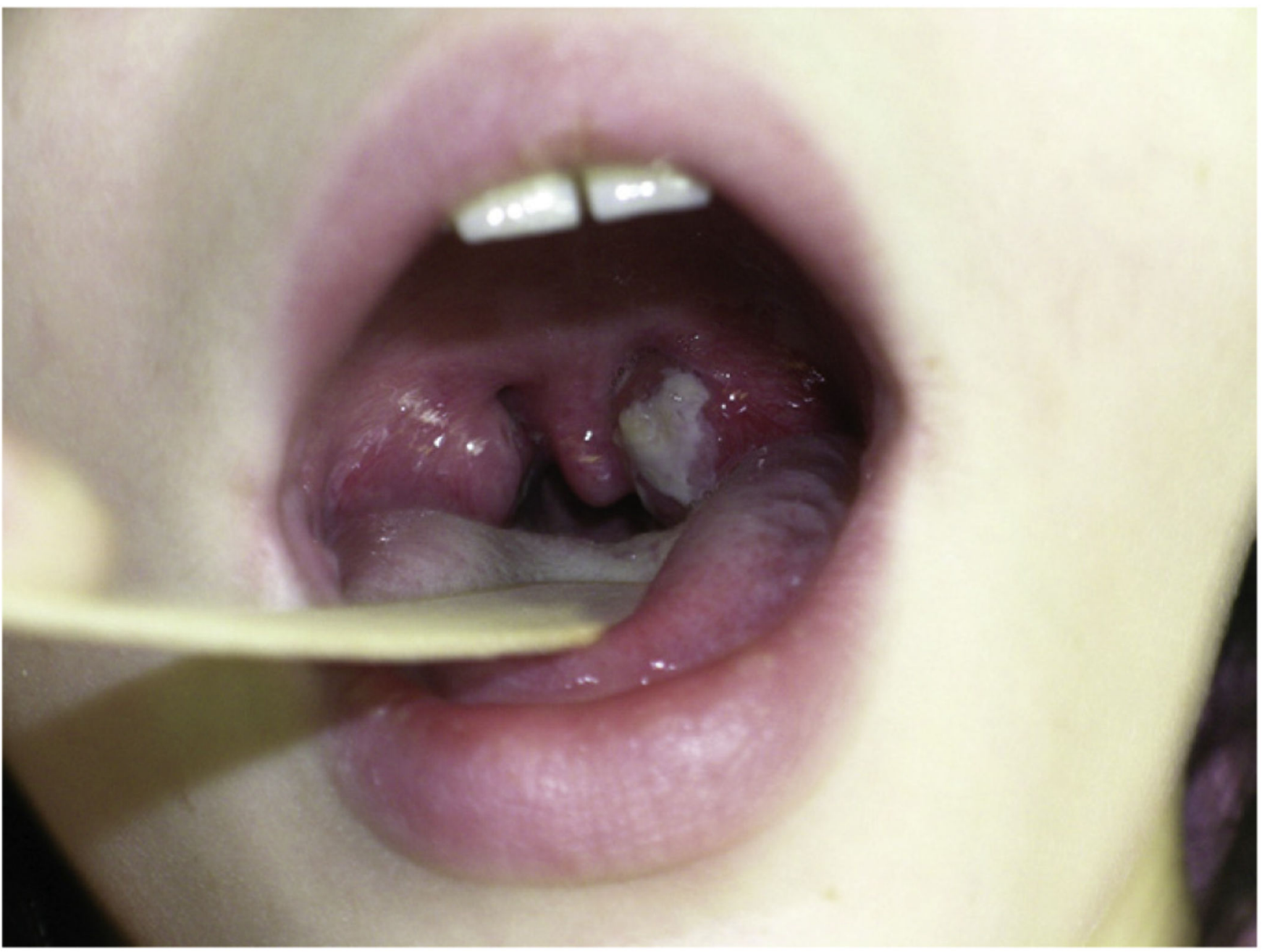

Figure 4.

Acute tonsillitis with exudates on the surface of the tonsils. 


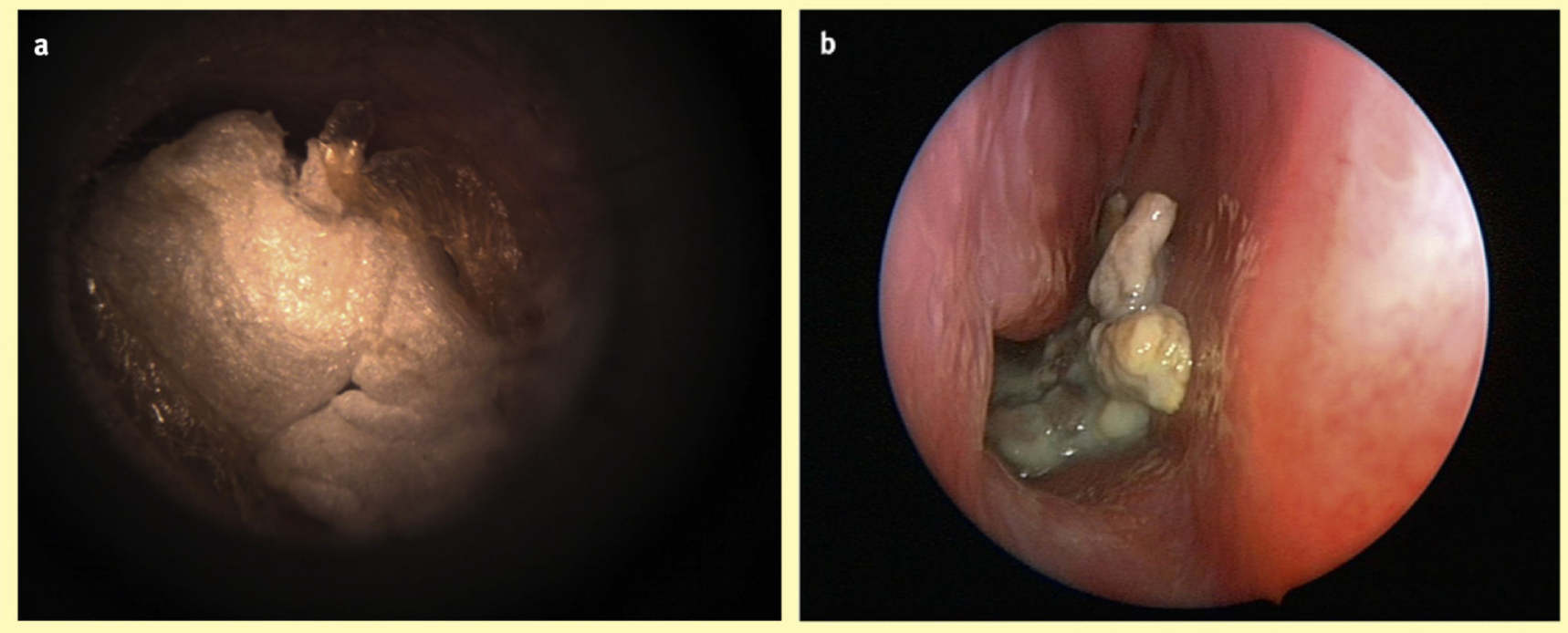

Figure 5.

(a) A piece of polystyrene in a child's ear. (b) A rhinolith in the right nasal cavity. 